\title{
The Linear Canonical Transform and Time-Frequency Representations
}

\author{
Qiang Xiang ${ }^{1, a}$ \\ ${ }^{1}$ College of Electrical\&Information ,Southwest University for Nationalities, Chengdu 610041,China \\ axqiang_0426@163.com
}

Keywords: Linear canonical transform; Wigner distribution; Time-frequency representation; Short-time Fourier transform

\begin{abstract}
The linear canonical transform(LCT), which is a generalization of the classical Fourier transform and fractional Fourier transform(FRFT), was introduced a number of years ago in the mathematics literature but appears to have remained largely unknown to the signal processing community, to which it may, however, be potentially useful. In this paper, we briefly introduce the LCT and a number of its properties and then discuss the LCT's relationships with time-frequency representations such as the Wigner distribution and the short-time Fourier transform. These relationships have a very simple and natural form and show that the LCT performs a homogeneous linear mapping in the time-frequency plane. Finally, an example of the application of the LCT is given.
\end{abstract}

\section{Introduction}

Fourier transform(FT) is one of the most frequently used tools in signal processing and is frequently used in many other scientific disciplines. The fractional Fourier transform(FRFT) [1-2] is the generalization of the FT. The FRFT also is an important signal processing tool. It can analyze the signal in between the time and frequency domains.

The FRFT can be further generalized into the linear canonical transform(LCT) [3-4].The LCT is a four parameter $(\mathrm{a}, \mathrm{b}, \mathrm{c}, \mathrm{d})$ class of linear integral transforms. It is also called the special affine Fourier transform or ABCD transform[5]. In the mathematics literature, the LCT was proposed some years ago. Although potentially useful for signal processing applications [4,6-10], this transform appears to have remained largely unknown to the signal processing community. With the development of time-frequency analysis, many time-frequency representations(TFR's) of signals, such as the short-time Fourier transform (STFT) [9], the spectrogram [9], the Wigner distribution (WD)[9] , the ambiguity function[9], etc, have been developed and often used in time-frequency signal processing. As a more generalized signal processing tool, we want to know LCT's relationships with TFR's. So the main purpose of this paper is twofold: First, to briefly introduce the LCT and its main properties and, second, to discuss the relationships between the LCT and several TFR's. Finally we present an example of an application about this transform.

\section{The LCT and its properties}

The LCT with parameters $(a, b, c, d)$ of a signal $f(t)$ is defined as [3]

$$
F_{(a, b, c, d)}(u)=L_{F}^{(a, b, c, d)}[f(t)]=\left\{\begin{array}{c}
\int_{-\infty}^{+\infty} f(t) K_{(a, b, c, d)}(t, u) d t, b \neq 0 \\
\sqrt{d} e^{j \frac{d}{2} u^{2}} f(d u), b=0
\end{array},\right.
$$

where parameters $(a, b, c, d)$ are real numbers satisfying $a d-b c=1$. The transformation kernel of 
LCT is $K_{(a, b, c, d)}(t, u)$, where

$$
K_{(a, b, c, d)}(t, u)=\sqrt{\frac{1}{j 2 \pi b}} e^{j\left(\frac{d}{2 b} u^{2}-\frac{1}{b} u t+\frac{a}{2 b} t^{2}\right)} .
$$

In this paper, only $\mathrm{b} \neq 0$ is taken into account, because LCT changes into the scaling operator when $\mathrm{b}=0$. The LCT is a unitary transform and has many special cases. When parameters $(a, b, c, d)$ $=(0,1,-1,0)$, the LCT coincides with the conventional Fourier transform. When $(a, b, c, d)=$ $(\cos \theta, \sin \theta,-\sin \theta, \cos \theta)$, the LCT coincides with the fractional Fourier transform. When $(a, b, c, d)$ $=(1, b, 0,1)$, the LCT reduces to the Fresnel transform (FRST) [4].

The LCT has the following properties, which will be useful later in this paper. it is easy to see that the LCT satisfies the additivity property as

$$
L_{F}^{(a, b, c, d)}[f(t)]=L_{F}^{\left(a_{2}, b_{2}, c_{2}, d_{2}\right)}\left[L_{F}^{\left(a_{1}, b_{1}, c_{1}, d_{1}\right)}[f(t)]\right] \text {, Where }\left[\begin{array}{ll}
a & b \\
c & d
\end{array}\right]=\left[\begin{array}{ll}
a_{2} & b_{2} \\
c_{2} & d_{2}
\end{array}\right] \cdot\left[\begin{array}{ll}
a_{1} & b_{1} \\
c_{1} & d_{1}
\end{array}\right] .
$$

It can be easily shown from the definition (1) that the LCT obeys the following modulation relation [4]:

$$
\begin{aligned}
& L_{F}^{(a, b, c, d)}[f(t-\tau)]=e^{j\left(u c \tau-\frac{a c}{2} \tau^{2}\right)} F_{(a, b, c, d)}(u-a \tau) \\
& L_{F}^{(a, b, c, d)}\left[f(t) e^{j \omega t}\right]=e^{j\left(u d \omega-\frac{d b}{2} \omega^{2}\right)} F_{(a, b, c, d)}(u-b \omega) .
\end{aligned}
$$

We also easily conclude that the inverse of an LCT with parameters $(a, b, c, d)$ is the LCT with parameters $(d,-b,-c, a)$ :

$$
f(t)=L_{F}^{(d,-b,-c, a)}\left[F_{(a, b, c, d)}(u)\right]=\left\{\begin{array}{c}
\int_{-\infty}^{+\infty} F_{(a, b, c, d)}(u) K_{(d,-b,-c, a)}(u, t) d u, b \neq 0 \\
\sqrt{a} e^{-j \frac{c a}{2} t^{2}} f(a t), b=0
\end{array}\right.
$$

From (6), we can see that the LCT consists of expressing $f(t)$ on a basis formed by the set of functions $K_{(d,-b,-c, a)}(u, t)$ (with $u$ acting as a parameter for spanning the set of basis functions). This basis is orthonormal and the basis functions are chirps, i.e., complex exponentials with linear frequency modulation.

The Parseval relation

$$
\int_{-\infty}^{+\infty} f(t) g^{*}(t) d t=\int_{-\infty}^{+\infty} F_{(a, b, c, d)}(u) G_{(a, b, c, d)}^{*}(u) d u
$$

is easily derived by expressing $f(t)$ in the left-hand side of this equation as the inverse transform of $F_{(a, b, c, d)}(u)$. A consequence of this equality is the energy-preserving property of the LCT

$$
\int_{-\infty}^{+\infty}|f(t)|^{2} d t=\int_{-\infty}^{+\infty}\left|F_{(a, b, c, d)}(u)\right|^{2} d u
$$

The Parseval relation and the energy-preserving property can also be viewed as consequences of the fact that the LCT is based on an set of orthonormal basis functions. Due to the energy-preserving property of the Fourier transform, the squared magnitude of the Fourier transform of a signal $|F(\omega)|^{2}$ is often called the energy spectrum of the signal and is interpreted as the distribution of the signal's energy among the different frequencies $e^{\text {jot }}$.Although it is less intuitive,(8) also allows us to call $\left|F_{(a, b, c, d)}(u)\right|^{2}$ the linear canonical energy spectrum of the signal $f(t)$, with parameters $(a, b, c, d)$, and to interpret it as the distribution of the signal's energy among the different chirps $K_{(d,-b,-c, a)}(u, t)$.

\section{The LCT's relationships with TFR's}

Relationships with the Wigner distribution. The Wigner distribution (WD) [9] is a very important time-frequency analysis tool, with applications in such diverse areas as radar and quantum mechanics. 
Apart from its direct applications, its importance also stems from the fact that many time-frequency analysis tools can be expressed in terms of the WD. We will now see that the WD has a simple relationship with the LCT. The WD of a signal $f(t)$ is defined as[6]

$$
W_{f}(t, \omega)=\int_{-\infty}^{+\infty} f\left(t+\frac{\tau}{2}\right) f^{*}\left(t-\frac{\tau}{2}\right) e^{-j \omega \tau} d \tau
$$

Let $W_{F_{(a, b, c, d)}}(u, v)$ be the Wigner distribution of $F_{(a, b, c, d)}(u)$, it can be shown [6] that the Wigner distribution of $F_{(a, b, c, d)}(u)$ is related to that of $f(t)$ by

$$
W_{f}(t, \omega)=W_{F_{(a, b, c, d)}}(a t+b \omega, c t+d \omega)
$$

The meaning of Eq. 10 is that the LCT performs a homogeneous linear mapping in the Wigner domain :

$$
\left(\begin{array}{l}
u \\
v
\end{array}\right)=\left(\begin{array}{ll}
a & b \\
c & d
\end{array}\right)\left(\begin{array}{l}
t \\
\omega
\end{array}\right)
$$

Examples of the linear mapping performed by some special case of LCT are depicted in Fig.1. The linear mapping preserves the area of the support in Wigner domain implying the conservations of degrees of freedom under LCT, which is consistent with the fact that the LCT is invertible. Many quadratic TFR's can be computed from the WD. The relationships of the LCT with these TFR's can normally be obtained from their definitions in terms of the WD and from the fact that the LCT induces a linear mapping in the WD. For example, it can be easily shown that the LCT also induces a homogeneous linear mapping in the ambiguity function.

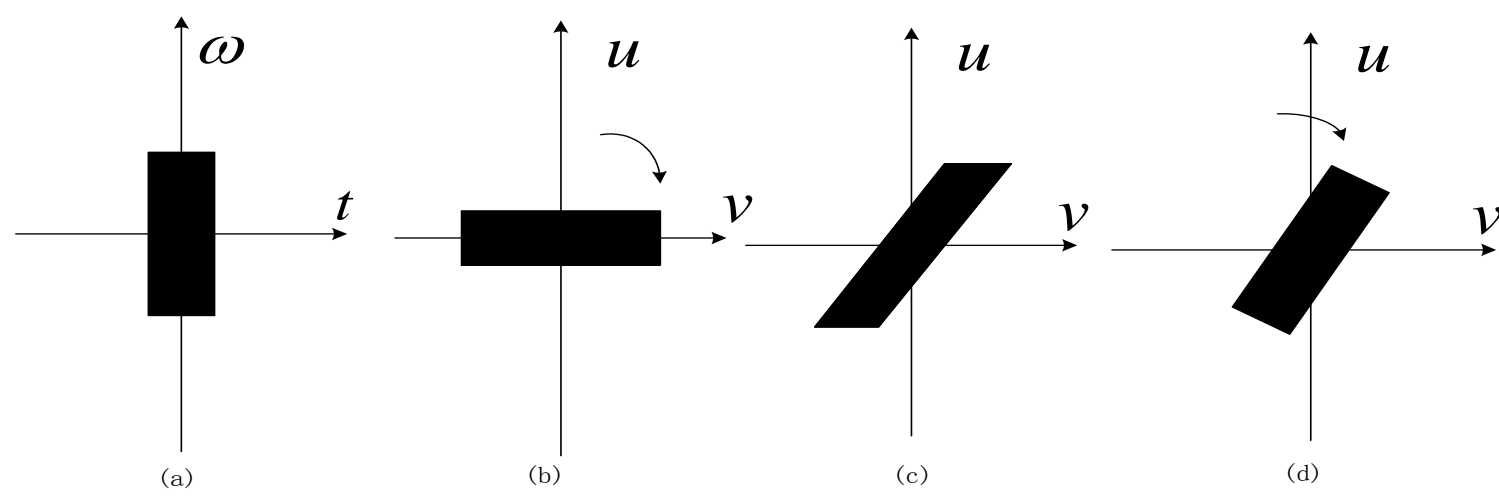

Fig.1 Examples of the effect of the LCT on the support of WD: (a) WD of the original signal,(b) FT, (c) FRST, (d) FRFT.

Relationships with the short-time Fourier transform. The short-time Fourier transform (STFT) is another important time-frequency analysis tool. It is frequently used, e.g., for speech processing. A related tool is the spectrogram, which corresponds to the squared magnitude of the STFT. The STFT of a signal $f(t)$ is defined as [1]

$$
S_{f}(t, \omega)=\frac{1}{\sqrt{2 \pi}} \int_{-\infty}^{+\infty} f(\tau) g^{*}(\tau-t) e^{-j \omega \tau} d \tau
$$

where $g(t)$ is a suitably chosen analysis window. The STFT can also be computed in a simple way from the Fourier transform of $f(t)$ :

$$
S_{f}(t, \omega)=\frac{1}{\sqrt{2 \pi}} e^{-j \omega t} \int_{-\infty}^{+\infty} F(v) G^{*}(v-\omega) e^{j v t} d v
$$

where $F$ and $G$ are the Fourier transforms of $f$ and $g$, respectively. This equation is similar to (12),except for the presence of the exponential factor $e^{-j \omega t}$.Now we define a modified short-time Fourier transform (MSTFT)[1], which consists simply of the normal STFT with a suitable phase modification: 


$$
\tilde{S}_{f}(t, \omega)=\frac{1}{\sqrt{2 \pi}} e^{j \frac{\omega t}{2}} \int_{-\infty}^{+\infty} f(\tau) g^{*}(\tau-t) e^{-j \omega \tau} d \tau
$$

From (13), the MSTFT can also be computed as

$$
\tilde{S}_{f}(t, \omega)=\frac{1}{\sqrt{2 \pi}} e^{-j \frac{\omega t}{2}} \int_{-\infty}^{+\infty} F(v) G^{*}(v-\omega) e^{j v t} d v
$$

Which is now very similar to (14),with no extra exponential factor. The MSTFT is, in this respect, more symmetrical than the STFT. To obtain the relationship between the LCT and the MSTFT, we will start from (14), replacing $f(\tau)$ by the LCT of $F_{(a, b, c, d)}$ with parameters $(d,-b,-c, a)$ :

$$
\begin{aligned}
\tilde{S}_{f}(t, \omega) & =\frac{1}{\sqrt{2 \pi}} e^{j \frac{\omega t}{2}} \int_{\infty}^{+\infty} \int_{\infty}^{+\infty} F_{(a, b, c, d)}(z) K_{(d, b,-c, a)}(z, \tau) d z g^{*}(\tau-t) e^{-j \omega \tau} d \tau \\
& =\frac{1}{\sqrt{2 \pi}} e^{j \frac{\omega t}{2}} \int_{\infty}^{+\infty} F_{(a, b, c, d)}(z) \int_{\infty}^{+\infty} K_{(a, b, c, d)}^{*}(\tau, z) g^{*}(\tau-t) e^{-j \omega \tau} d \tau d z
\end{aligned}
$$

The inner integral is the complex conjugate of the LCT with parameters $(a, b, c, d)$ of $g(\tau-t) e^{j \omega \tau}$, taken as a function of $\tau$ and with argument $z$.Using Eq. 4 and Eq. 5, we can conclude that this integral is equal to

$$
G_{(a, b, c, d)}^{*}(z-a t-b \omega) e^{j\left(\frac{a c}{2} t^{2}+\frac{b d}{2} \omega^{2}-z d \omega-z c t+b c \omega t\right)}
$$

and therefore

$$
\tilde{S}_{f}(t, \omega)=\frac{1}{\sqrt{2 \pi}} e^{j \frac{\omega t}{2}} \int_{-\infty}^{+\infty} G_{(a, b, c, d)}^{*}(z-a t-b \omega) \times F_{(a, b, c, d)}(z) e^{j\left(\frac{a c}{2} t^{2}+\frac{b d}{2} \omega^{2}-z d \omega-z c t+b c \omega t\right)} d z
$$

We now make, on the right-hand side, the change of variables from $(t, \omega)$ to $(u, v)$, corresponding to a coordinate transformation as $u=a t+b \omega, v=c t+d \omega$, and after simplification of the exponents, we obtain

$$
\tilde{S}_{f}(t, \omega)=\frac{1}{\sqrt{2 \pi}} e^{j \frac{u v}{2}} \int_{-\infty}^{+\infty} F_{(a, b, c, d)}(z) \times G_{(a, b, c, d)}^{*}(z-u) e^{-j z v} d z
$$

The right-hand side of this equation is the MSTFT of $F_{(a, b, c, d)}(u)$ computed with window $G_{(a, b, c, d)}(u)$ and with arguments $(u, v)$.The left-hand side is the MSTFT of $f(t)$ computed with window $g(t)$ and with arguments $(t, \omega)$.As in the case of the WD, the LCT also performs a homogeneous linear mapping in the MSTFT domain.

The spectrogram is simply the squared magnitude of the STFT and, therefore, of the MSTFT as well. The results we obtained on the MSTFT immediately lead us to conclude that the effect of the LCT on the spectrogram is identical to the one it has on the MSTFT: the spectrogram of $F_{(a, b, c, d)}(u)$ computed with window $G_{(a, b, c, d)}(u)$ is a linear mapping of the spectrogram of $f(t)$ computed with window $g(t)$.

\section{Application}

In this section, we will briefly present, as an example, the application of the LCT to the study of swept-frequency filters[1]. These filters are used, for example, in frequency analyzers for high frequency signals. Swept-frequency filters are linear time-varying systems that can be represented in the form shown in Fig.2. The output of the swept-frequency filter is given by

$$
y(t)=\left[\left(f(t) e^{-j \frac{c}{2} t^{2}}\right) * g(t)\right] \cdot e^{j \frac{c}{2} t^{2}}=e^{j \frac{c}{2} t^{2}} \cdot \int_{-\infty}^{+\infty} f(\tau) e^{-j \frac{c}{2} \tau^{2}} g(t-\tau) d \tau
$$

Where $g(t)$ is the impulse response of the shift-invariant filter in Fig.2. 


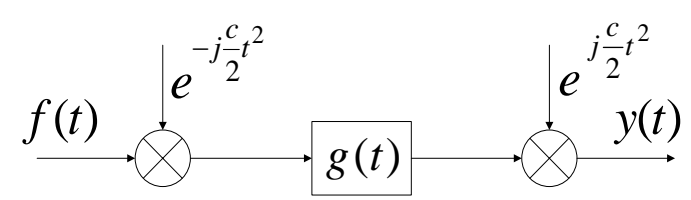

Fig.2 Representation of a swept-frequency system.

Let us compute the LCT of $y(t)$, choosing parameters $(a, b, c, d)=(-c, 1,-1,0)$ :

$$
\begin{aligned}
Y_{(-c, 1,-1,0)}(u) & =\sqrt{\frac{1}{j 2 \pi}} \int_{-\infty}^{+\infty} \int_{-\infty}^{+\infty} e^{-j u t} e^{-j \frac{c}{2} t^{2}} f(\tau) e^{j \frac{c}{2}\left(t^{2}-\tau^{2}\right)} g(t-\tau) d \tau d t \\
& \left.=\sqrt{\frac{1}{j 2 \pi}} \int_{-\infty}^{+\infty} e^{-j \frac{c}{2} \tau^{2}} f(\tau) \int_{-\infty}^{+\infty} g(t-\tau) e^{-j u t} d t\right) d \tau=\sqrt{\frac{1}{j 2 \pi}} \int_{-\infty}^{+\infty} e^{-j \frac{c}{2} \tau^{2}} f(\tau) e^{-j u \tau} G(u) d \tau \\
& =F_{(-c, 1,-1,0)}(u) \cdot G(u)
\end{aligned}
$$

Where $G(u)$ is the (classical) Fourier transform of the impulse response $g(t)$. Therefore, $G(u)$ can be called the transfer function of the swept-frequency filter in the LCT domain. The use of the LCT and of this transfer function allows a treatment of swept-frequency filters that is very similar to the classical treatment of time-invariant filters with the Fourier transform.

It is also worth mentioning that the LCT is useful for solving certain classes of differential equations that appear, for example, in quantum mechanics and optics, and that may also be useful in signal processing applications.

\section{Conclusion}

We have presented an extension of the Fourier transform and fractional Fourier transform, which is designated linear canonical transform. This linear transform depends on parameters $(a, b, c, d)$ and can be used to convert a signal from the time domain to the LCT domain. The LCT is a unitary transform. Its special cases, such as the FT, the FRFT and the FRST are widely used in various fields.

In this paper, the LCT was shown to correspond to a representation of the signal on an orthonormal basis formed by chirps. On the other hand, the LCT was also shown to induce a linear mapping in various time-frequency transforms, including the Wigner distribution and the short-time Fourier transform. The relationships between the LCT and time-frequency transforms suggest that it can be a useful tool to further study the properties of these transforms and to develop time-frequency transforms better suited to specific applications. Finally, an application example was given, showing how the use of the LCT allows a treatment of swept-frequency filters that is very similar to the classical treatment of shift-invariant filters with the Fourier transform.

\section{Acknowledgements}

This work is supported by Scientific Research Fundation of the Education Department and Science\&Technology Department of Sichuan Province, China(No. 14ZA0369, 2014GZ0015)

\section{References}

[1] L. B. Almeida, The fractional Fourier transform and time-frequency representations. IEEE Trans Signal Processing, Vol.42(1994)No.11, p.3084.

[2] H. M. Ozaktas, M. A. Kutay, and Z. Zalevsky, The Fractional Fourier Transform With Applications in Optics and Signal Processing. New York: Wiley (2000).

[3] M. Moshinsky, C. Quesne. Linear canonical transformations and their unitary representations. J 
Math Phys, Vol.12(1971)No.8, p.1772.

[4] B.Z. Li, R.Tao, Y. Wang. New sampling formulae related to linear canonical transform[J]. Signal Processing, Vol.87(2007)No.5, p.983.

[5] Qiang Xiang, Kai-Yu Qin. Convolution, correlation, and sampling theorems for the offset linear canonical transform, Signal, Image and Video Processing,Vol.8(2014)No.3, p.433.

[6] S.C. Pei, and J.J. Ding, Relations between fractional operations and time-frequency distributions, and their applications.IEEE Trans.Sig.Proc. ,Vol.49(2001)No.8,p. 1638.

[7] Kamalesh Kumar Sharma,Shiv D.J., Signal separation using linear canonical and fractional Fourier transforms. Opt Communications, Vol.265(2006)No.2, p. 454.

[8] B. Barshan, M. A. Kutay, H. M. Ozalctas., Optimal filters with linear canonical transformations. Opt Communications, Vol.135(1997), p.32.

[9] F. Hlawatsch and G. F. Boudreaux-Bartels, Linear and quadratic time-frequency signal representation, IEEE Signal Processing Magazine, Vol.9(1992)No.2, p.21.

[10]A. Stern. Sampling of linear canonical transformed signals[J]. Signal Processing, Vol.86 (2006) No.7,p.1421. 